Yazdani, N, Yazdani, F and Nobakht, L

"Reflective self-awareness exercise; steps towards changing students' attitudes towards disability".

Yazdani, N, Yazdani, F and Nobakht, L (2016) "Reflective self-awareness exercise; steps towards changing students' attitudes towards disability".International Journal of Therapies and Rehabilitation Research, 5 (2). pp. 25-29.

doi: $10.5455 /$ ijtrr.000000122

This version is available: https://radar.brookes.ac.uk/radar/items/4e003aa8-eaf0-4338-9fcf-750a667a0f3d/1/

Available on RADAR: March 2016

Copyright (C) and Moral Rights are retained by the author(s) and/ or other copyright owners. A copy can be downloaded for personal non-commercial research or study, without prior permission or charge. This item cannot be reproduced or quoted extensively from without first obtaining permission in writing from the copyright holder(s). The content must not be changed in any way or sold commercially in any format or medium without the formal permission of the copyright holders.

This document is the published version of the journal article. 


\title{
Reflective self-awareness exercise; steps towards changing students' attitudes towards disability Nastran Yazdani ${ }^{1}$, Farzaneh Yazdani ${ }^{2}$, Laya Nobakht ${ }^{3}$
}

\author{
${ }^{1}$ Researcher, Al Zahra University, Iran \\ ${ }^{2}$ Senior Lecturer, Oxford Brookes University, UK \\ ${ }^{3}$ MSc Clinical Psychology student, Azad University, Iran \\ Correspondence: Nastran Yazdani, email:fyazdani@bookes.ac.uk
}

\begin{abstract}
The aim of this study was to explore the changes in student's attitudes regarding disability after attending the module 'Disability: theories, nature and experiences' designed around reflective self-awareness exercises. Literature indicates enhanced knowledge does not automatically lead to changes in attitude. Health care professionals are the most significant people to instil hope in their clients to pursue a happy life. As advocates for people with disability, health care professionals need themselves to believe that people with disability can pursue a happy life as abled bodied people do. Researchers aimed to explore the impact of the 'Disability' module using discussion and reflective exercises, on students' way of thinking about and changes in attitude towards disability. Students were asked to write stories at the beginning and after completing the module. A thematic analysis was applied to identify the students' way of communicating their thoughts and feelings about disable bodied/disability before and after the module. Three major themes were identified to represent the differences before and after attending the module: problem /solution oriented approach towards perceived problems, separating/ integrating disable/able bodied, passive/ active role of disable bodied and society.
\end{abstract}

Key words: Thematic analysis; Qualitative study; Reflection; Rehabilitation

\section{Introduction}

The attitude of health care professionals has the potential to greatly improve or hinder opportunities for persons with disabilities to be fully included in their communities. Negative attitudes of health care professionals are considered to be an invisible barrier to rehabilitation and integration. In contrast, positive attitudes are a key to successful integration (AlAbdulwahab \& Al-Gain 2003). Findings from the study carried out by Au and Man (2006) demonstrated that more negative attitudes towards disability were shown by students compared to health care professionals. Accordingly recommendations were made to modify the training curriculum and enhance the quality of services to develop more favourable attitudes towards people with disabilities.A study done by Katz and Hayout (2002) demonstrated the positive impact that special attention in education can have on developing positive attitudes towards disabilities.

A common problem with attitude research is that respondents may provide socially sanctioned or politically correct responses to questions about attitudes. Social desirability response style is a problem of using direct methods of attitude measurement (Taylor, 1961). According to Jung (1971) attitude is a "readiness of the psyche to act or react in a certain way" (Main 2004, p 40). Attitudes, therefore could be measured explicitly through self- reports or observed behaviours. Implicit measures however, usually rely on an indirect measure of attitude. Implicit measures are not consciously directed and are assumed to be automatic, which may make implicit measures more valid and reliable than explicit measures (Fazio and Olson, 2003). Much of attitude research has emphasized the importance of affective or emotional components. Emotion works hand-in-hand with the cognitive process, or the way we think, about an issue or situation. The multicomponent model is the most influential model of attitude. Bem (1970) states that attitude consists of cognitive, affective, and behavioural components. The cognitive component of attitudes refers to the beliefs, thoughts, and attributes that we would associate with an object. A person's attitude might be based on the negative and positive attributes they associate with an object. The affective component of attitudes refers to feelings or emotions towards the object of attitude. The behavioural component of attitudes refers to past behaviours or experiences regarding the object.

This study used an indirect method of studying attitude which was through students' stories about disability and disable bodies hoping to capture implicit or unconscious elements of their attitudes. There is a strong emphasis on improving selfawareness of health care professionals in relation to sensitive issues such as the culture of minority groups including people with disabilities (Comas-Diaz, 2012). The module 'Disability 
theories, nature and experiences' is one of the modules within the MSc Rehabilitation course at Oxford Brookes University. Critical self-awareness, professional and personal development, valuing human diversity and global citizenship are emphasized as graduate attributes. The learning objectives for this module include enhancing students self-awareness and reflection on their attitudes towards disability. The module applies several activities to support students to achieve these. Reflection has been used widely in education to enhance the quality of learning (Bulman, \& Schutz, 2013) and was used as an educational strategy within this module. The educational strategies were planned to facilitate students integrating their past experiences, current knowledge and feelings about the issue of disability.

On previous runs of the module, students were required to submit a reflection on their learning to complete the module. These reflections had shown that students thought the module had changed their thinking and ideas about disability; cognitive aspects that are explicitly presented. The module leader was interested to learn whether the students' reflections submitted on completing the module were socially sanctioned and politically correct responses. Therefore, this study aimed at exploring attitude and changes in attitude towards disability before and after attending the 'Disability' module using student's stories in order to capture implicitly expressed components of attitude.

\section{Methods}

Once enrolled on the module 'Disability theories, nature and experiences', an invitation letter, information sheet, and consent form were sent to students. All of the students were first asked to take part in an activity to write a story about a person with a disability. It was left to students to think about what to include in their story. Students were asked to submit another story about a person with a disability at the end of the semester. The task was done in the classroom as part of the module activities, but students could identify in the consent form whether they agreed to their activity to be analysed for research purposes. As part of the invitation letter students were assured that this would not impact on their final evaluation and they could attend the first task and not the second one if they wished to. Students were informed that the study had university ethics committee approval. They all agreed to put their name to their work. Therefore, it was possible to compare each student's stories before and after the module. This article focuses on the overall findings from each task and the findings (themes) are based on similarities and differences between the two. Students were also asked to complete a reflection at the end of the module. The aim was to examine the students' self- awareness in relation to changes in their thinking and feeling about disability and disabled people. The rationale was that while reflection represents students' awareness about changes as they perceive them, stories would identify some deeper attitudes towards disability which might not be at the students' conscious level. As participants had put their name against their stories as well as their reflection, with their agreement researchers were able to compare the final student reflection on their learning process through the module with the stories.

\section{i) Methodology, conceptual frame work}

Encouraging and facilitating enquiry, critical ((Pithers \& sodden 2000) and creative thinking (Devidovich a\& Milgram 2006), selfawareness (Dochy, Segers, Sluijsmans 1999) and reflection and story-telling (Alterio \& McDrury 2003) have been shown to be successful education strategies. These strategies are pulled together to support students' learning and change towards a more realistic and humanistic approach towards disability. This study uses a qualitative approach, to explore the changes in students' views of disability. Qualitative methodology was felt to be the most suitable approach for studying students' feeling and thinking, represented in their words, narratives and the way they express them ( Creswell 2013).

\section{ii) Setting and time scale}

The module runs in the second semester of a one-year MSc program at the Oxford Brookes University. There were 6 seminars and lectures of about 5 hours, every other week. A part of the learning objectives of this module is establishing a positive attitude towards disability and working with people with disability. The whole period of the module is 12 weeks plus a week for the assignment.

\section{ii) Participants}

Participants were MSc Rehabilitation students with different professional backgrounds and diverse nationalities (India, Britain, Kenya, Italy, Brazil, Saudi Arabia, Mali).

Table 1. Students' professional background

\begin{tabular}{|l|c|c|}
\hline \multirow{2}{*}{ Profession } & \multicolumn{2}{|c|}{ Number of Students } \\
\cline { 2 - 3 } & Before & After \\
\hline Social Worker & 3 & 3 \\
\hline Physiotherapist & 7 & 6 \\
\hline Medical Doctor & 1 & 1 \\
\hline $\begin{array}{l}\text { Occupational } \\
\text { therapist }\end{array}$ & 2 & 2 \\
\hline Nurse & 2 & 2 \\
\hline Physical Educator & 1 & 0 \\
\hline
\end{tabular}

\section{i) Ethical approval for the study}

The study was approved by the ethics committee, Oxford Brookes University.

\section{iv) Intervention}

The content of the module was as follows: History of discrimination and disabled people, Disability definition and global perspective, Introduction to models of Disability, Disability, attitude and culture, the Voice of people with disability; special issues such as sexuality, Research issues related to disability, The politics of disability, Disability rights.

Students had to prepare before coming to each session. Questions were set to encourage students to prepare for the 
following seminar. The question could be based on a video, case scenario and the like. Here are presented some of these questions:

What does disability mean to me?

What is the politics of the meaning of disability?

How would the definition of disability impact the person with disability and others?

How would you handle the paradox of improving clients' capacities while valuing disability as diversity?

What do you think about sex workers who provide services for people with disability?

Each week included a lecture followed by a seminar facilitating students' discussion and group work. Some examples of the activities and discussion group work are as follows:

Students to interview up to 3 people with diversity in age, gender, nationality, religious beliefs etc about the way they see disability. For the first part of this activity students were asked to think about the way they would come up with the most genuine response from their interviewees. Students could choose a variety of modes such as communicating with their interviewee via a picture, stories, or direct dialogue about the topic. They could choose skype, phone or face to face interview.

Each seminar ended with a guided reflective discussion exercise in the classroom. Students were then invited to post their thoughts on the seminar topic on the online discussion board, and encouraged to respond to other postings and enter into discussion with each other. Students also had the option to post their more intimate thoughts and feelings on a personal learning log, seen only by themselves and the module leader. This gave the students the chance to record their thoughts and feelings about disability and people with disability as they progressed through the module.

An example of these structured reflective exercises was reflecting on their feelings after watching a video about a person with disability. Examples of these videos chosen from different sources were:

"Ugliest Woman In The World -- Lizzie Velasquez", "The Family that Walks on All Fours", "What's disability to me"; videos from WHO websites, several of other videos from Oxford Brookes University pool of educational resources including real narratives of health care service users. These videos were selected to illustrate the issues that peoples with disability face, their personal and subjective experiences of impairment and their interpretation of their needs. As part of their activities students were asked to find narratives from people with disabilities, and post these on the discussion forum, along with the students' own analysis of these stories, to establish a point of discussion.

The module introduces a variety of approaches to how disability is viewed, interpreted and addressed through medical, biopsychosocial, affirmative, and social models. Students are encouraged to adopt a critical view of these approaches. This also includes reflection on how they keep a balance between their role as therapist on the one hand, providing services to enable a return to a 'normal' and independent life, and their role as a student, reflecting on the person's subjective experience of disability, on the other. This paradox, working at the interface of medical and social models, seeing disability as both something to be treated and also as a form of human diversity that must be understood within its social context, lies at the heart of the therapeutic alliance with patients (Roush \& Sharby 2011).

The following exercises were planned to provide an environment for better understanding what is really like to have an impairment that is disabling and within a community of people with different attitudes and beliefs about this issue. An example was reviewing the report of a survey done in the UK 2009: Public Perceptions of Disabled People Evidence from the British Social Attitudes, and discussing the findings in their groups. The document included the following research reports.++++

The final assignment of the module was also developed to provide students with an opportunity to raise awareness about a topic that they believe is not being addressed in their own country or practice context. They were asked to develop an information aid to heighten people's awareness of disability. It could be aimed at professionals, clients or carers and could be in the form of a poster presentation, booklet, video, planned talk, resource. Here are some examples of students' final assignments that represent how their learning in this module was reflected in their choice of topic.

- People with disabilities and driving: Raising awareness of their characteristics and the problems they face, addressing the public using a booklet

- Raising employers' awareness about people with multiple sclerosis; poster

- Problems that disabled children encounter in Physical Education lessons at school: Using a power point as an information aid to promote PE and sport activities among children with disabilities in school

v) Data analysis

Whilst participants were not anonymous to the first author initially, the data were de-identified for further analysis. The second author who was not related to the module initiated the first stage of analysis immediately after the first submission. 16 participants completed the first and 14 of them completed the second task. Thematic analysis was chosen as the best method to answer the research question of the study. Thematic analysis helps researchers to identify themes and patterns from what is observed or heard from people and aims to generate descriptions of strategies and behaviours (Hammersley \& Atkinson 1995).

Diagram 1 demonstrates the key codes, categories and themes. 


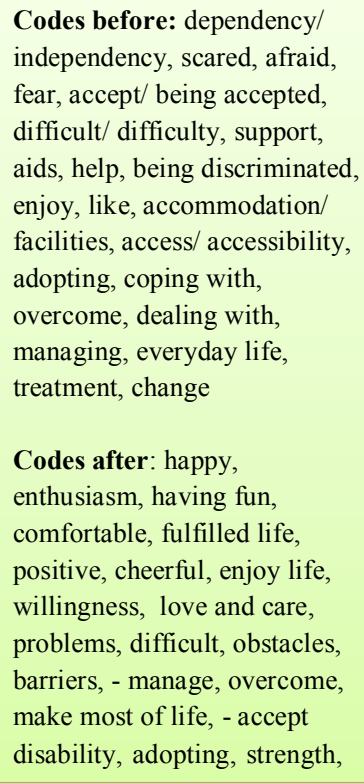

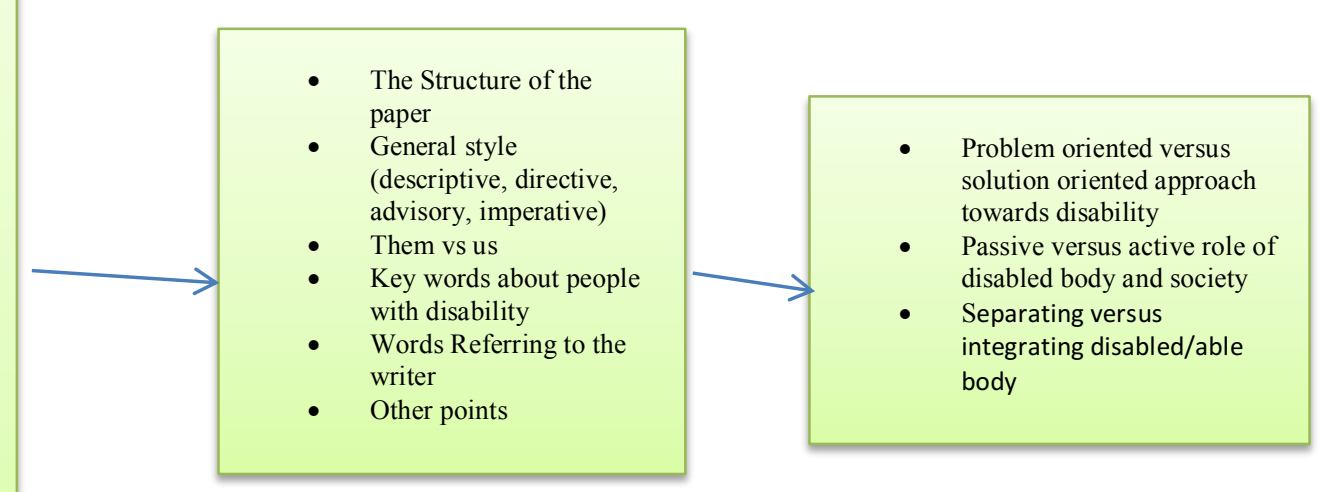

Thematic analysis was applied based on the manual by Saldana 2012. The key words and concepts were identified and compared in the before and after texts which led to the following themes.

\section{Results and Discussion}

The final three themes identified represent some of the changes in students' attitudes towards disability as a concept and people with disability.

Problem oriented versus solution oriented approach towards disability

Most stories demonstrated the thoughts and feelings of the person with disabilities. Within the first task, students showed more of a wishful thinking, or longing for lost opportunities. "...I (student as narrator) should be thankful for what I have", "She lost privacy, independence, very difficult time in her life", "I wish all disabled people could be strong...". The stories were more around the impairment and limitations caused by them. Most stories represented the narrators' understanding of the difficulties that people with disabilities face due to their impairment. "...these people have real difficulties..."

Stories showed mostly the sad feelings and frustrations of people with disabilities. Stories were more representative of the early stage of facing disabilities. On the other hand, the stories written after completing the module represented more the later stages of disabilities when acceptance had occurred and the character of the story was thinking of strategies to cope with their problems, and identifying strategies. "I (the narrator impersonates the person with disability) begin to think of returning home with a friend who will be moving in to help me". Stories presented more hope and some levels of strategic planning for the future. "... yes I've had a stroke, but I have gotten better, and I can still get better"

Passive versus active role of disabled body and society: The first task represented more of a medical approach to disability, emphasising impairment and limitations in function. The character was called 'disabled' mostly based on their impairment. A disabled person was a more passive person or victim in the first task, while in the second task not only the person but others such as family, friends and society were agents and acting to make a difference in the situation. “...I (narrator impersonates a person with disability) am aware of my problem...yes I have a problem, I get anxious but I am aware when my thoughts are not realistic...I feel I can cope with this anxiety on my own but am happy to seek support when needed, I won't be the victim of impairment! No... I am Samantha not a disabled"

There were more social elements discussed in the second task and participants showed more insight into disability rights and the role of society, government and law on disabling/ enabling situations. "...Knowing laws and rights I know things will fall into place..."

\section{Separating versus integrating disabled/able body}

The use of language seemed an interesting phenomenon in all the stories. While it seemed to be less of a problem for students to imagine themselves as a person with disability in the second task, in comparison some students had difficulties writing stories from the perspective of a person with disability in the first task. These students mostly referred to people with a disability as 'they' and themselves a narrator ' $w e^{\prime}$ ' or ' $I$ '. Negative verbs such as 'can' $t$ ', ' am not able' were more common in the first task while verbs with positive meaning such as 'manage', 'adapt', 'accept' were seen more in the second task. Absolute adjectives such as 'always' or 'everything' representing a black and white attitude could be seen in the first task but not in the second task. Instead adverbs such as 'sometimes', 'some people' were seen more in the second task. Words representing positive thinking, planning, hope, realism could be seen more in the second task. 
There were varieties of style in writing the stories. Within the first task some students showed an advisory or imperative approach in writing such as 'one with disability should do...'. However, this was only seen in one students' text in the second task and the rest were written mostly in an indirect style where the narrator had taken an active role as one with disability doing things.

It felt as if students were sympathising with people with disabilities and were going through the stages of grief in their stories and as the semester carried on they had more acceptance and had developed more belief in (their) disabled bodies.

\section{Discussion}

The findings of this study show that Master's students in the field of rehabilitation show a good level of understanding of the issues and difficulties people with disability are exposed to even before starting the Disability module. These students were health care professionals by background and had some experience of working with this population. This is congruent with the findings of a study carried out by Au and Man (2006). However, students' attitudes represented in their stories written before attending the module matched medical models which separate people with disabilities from able bodied people, emphasising impairment and treatment. Even though there was a high level of sympathy presented by students before attending the module, most students did not address the role of subjective experiences of disability in their stories. The module improved the students' understanding of disability as a phenomenon defined by the person's perceptions, society's opportunities and demands as well as the impairment.

Comparing the stories before and after through use of words, hidden messages, metaphors showed a sense of loss, with associated feelings of anger and sadness. Students expressed feelings of sadness for the disabled body, becoming emotional and wishful, feeling sorry for the person, not being able to think beyond the disability, thinking from the perspective of a therapist who wants to treat the impairment and improve function.

In the second story the students showed more acceptances and started to think of the disabled body as a person within society. The module obviously had an impact on the way students were dealing with disability issues which could be positive steps towards changing attitudes. The students had mentioned the importance of activities, reflective exercises as well as discussions in changes that they had observed in themselves in the final reflection and feedback about the module.

This study had limitations due to small sample size and were representative of the students' professional backgrounds. Therefore, the findings should be used as evidence with consideration of these limitations.

\section{Conclusions}

The findings of this study could be applied when educating health care students in relation to disability issues. Reflective practice is an important element in moving through stages of accepting, and understanding the disabled person's world. Students need time and appropriate exercises to develop a deeper understanding of the disabled person's life and themselves as health care professionals in relation to the issue of disability. This can lead to a more positive and reality-oriented approach towards the issue of disability and people who experience disability.

\section{Conflict of Interest: None.}

\section{References}

Al-Abdulwahab, SS., Al-Gain, SI. (2003). Disability among clients attending TAIF rehabilitation centre, Saudi Arabia. Asia Pacific Disability Rehabilitation Journal, 14 (1), 63-70.

Alterio M, McDrury J (2013) Learning through storytelling in higher education: Using reflection and experience to improve learning SAGE London

Au, KW. Man, DWK. (2006). Attitudes toward people with disabilities: a comparison between health care professionals and students. International Journal of Rehabilitation Research, 29 (2), 155-60.

Bem DJ (1972) Self Perception theory cited in Haddock and Maio (2004) Contemporary perspectives on psychology of attitudes, Hove and New York, Psychology press.

Bulman, C. \& Schutz, S. (2013). Reflective Practice in Nursing (5th ed.) Blackwell-Wiley, Oxford.

Comas-Diaz, L. (2012). Multicultural Care: A Clinician's Guide to Cultural Competence (Psychologists in Independent Practice Series). Washington DC. American psychological association.

Davidovitch N, Milgram RM (2006) Creative thinking as a predictor of teacher effectiveness in higher education. Creativity Research Journal, (1), 31-35. http://dx.doi.org/10.1037/a0016965

Dhingra, Y. Bhatnagar, V. (2006). Attitudes of speech-language pathology students towards persons with disability. Asia Pacific Disability Rehabilitation Journal, 17 (1), 94-100.

Dochy F, Segers M, Sluijsmans D (1999) The use of self-, peer and co-assessment in higher education: A review. Studies in Higher Education. 24(3), 331-350.

Fazio, R. H. \& Olson, M. A., (2003). Implicit Measures in Social Cognition Research: Their Meaning and Uses. Annual Review of Psychology. 54, 297-327

Honey, M. Waterworth, S. Baker, H. Lenzie-Smith, K. (2006). Reflection in the disability education of undergraduate nurses: an effective learning tool? Journal of Nursing Education, 45 (11), 449-53.

Katz, S. Hayout, I. (2002). Impact of an educational programme on nursing for children with developmental disabilities. British Journal of Developmental Disabilities, 48 (94), 27-37.

Kubler-Ross, E.(2009). On Death and Dying (40 th ed.). Abingdon. Routledge

Main, R. (2004). The rupture of time: Synchronicity and Jung's critique of modern western culture. Routledge. New York.

Paris, MJ. (1993). Attitudes of medical students and health-care professionals toward people with disabilities. Archives of Physical Medicine \& Rehabilitation, 74 (8), 818-25.

Pithers R.T. \& Soden R (2000) Critical thinking in education: a review Educational Research. 42(3): 237-249. Doi: 10.1080/001318800440579

Roush, SE, Sharby, N (2011) Disability reconsidered: the paradox of physical therapy. Physical Therapy, 91(12), 1715-1727.

Saldana, J. (2012). The Manual for Qualitative Researchers. London. SAGE.

Taylor, J. B. (1961). What do attitude scales measure: The problem of social desirability. The Journal of Abnormal and Social Psychology, 62(2), 386-390. 